\title{
Cognitive behavioral psychotherapeutic treatment at a psychiatric trauma clinic for refugees: description and evaluation
}

\author{
Cæcilie Buhmann, MD, PhD*, Ida Andersen, MSc*, Erik Lykke Mortensen, MSc**, \\ Jasmina Ryberg, MD*, Merete Nordentoft, DrMSc***, Morten Ekstrøm, MD, PhD*
}

\begin{abstract}
Introduction: Cognitive behavioural therapy (CBT) with trauma focus is the most evidence supported psychotherapeutic treatment of PTSD, but few CBT treatments for traumatized refugees have been described in detail.
\end{abstract}

Purpose: To describe and evaluate a manualized cognitive behavioral therapy for traumatized refugees incorporating exposure therapy, mindfulness and acceptance and commitment therapy.

Material and methods: 85 patients received six months' treatment at a Copenhagen Trauma Clinic for Refugees and completed self-ratings before and after treatment. The treatment administered to each patient was monitored in detail. The changes in mental state and the treatment components associated with change in state were analyzed statistically. Results: Despite the low level of functioning and high co-morbidity of patients, $42 \%$ received highly structured CBT, which was positively associated with all treatment outcomes. The more methods used and the

\footnotetext{
*) Competence Center for Transcultural Psychiatry (CTP), Psychiatric Center Ballerup, the Mental Health Services of the Capital Region of Denmark

$\star \star)$ Institute for Public Health, University of Copenhagen

$\star \star \star)$ Psychiatric Center Copenhagen, the Mental Health

Services of the Capital Region of Denmark
}

Correspondence to: caecilie.boeck.buhmann@regionh.dk more time each method was used, the better the outcome. The majority of patients were able to make homework assignments and this was associated with better treatment outcome. Correlation analysis showed no association between severity of symptoms at baseline and the observed change.

Conclusion: The study suggests that CBT treatment incorporating mindfulness and acceptance and commitment therapy is promising for traumatized refugees and punctures the myth that this group of patients are unable to participate fully in structured CBT. However, treatment methods must be adapted to the special needs of refugees and trauma exposure should be further investigated.

Keywords: Refugee, trauma, CBT, PTSD, depression

\section{Introduction}

Although evidence-based interventions exist for PTSD in the general population, treatment of PTSD among refugees is much less researched. At the trauma clinic for refugees, we are regularly confronted with other issues of central importance to our patients, such as depression, somatoform disorders, psychosis, chronic pain and personality disorders. Cultural differences between refugee populations and western 
populations for which most PTSD interventions are designed, further complicate the treatment.

A systematic review of randomized controlled trials of treatments for PTSD among refugees and asylum seekers concluded that no intervention form is currently firmly supported. ${ }^{1}$ However, several approaches seem promising. Firstly, significantly positive results of both cognitive-behavioural therapy and exposure therapy have been found in several studies. ${ }^{2,3}$ Secondly, culturally adapted cognitive-behavioural therapy has been successfully applied in the treatment of Cambodian refugees. ${ }^{4-6}$ Thirdly, there seems to be some success in the use of narrative exposure therapy, which has been used with refugees from Sudan, Somalia and Rwanda. ${ }^{7-9}$ A Cochrane review of evidencebased psychotherapy interventions for PTSD in the general population concludes that individual trauma focused cognitive-behavioural therapy (TFCBT), eye movement desensitization and reprocessing (EMDR), stress management and group TFCBT are effective in the treatment of PTSD. ${ }^{10}$ It highlights that trauma focused treatments are more effective than non-trauma focused treatments. The population in this study is described in detail elsewhere and the treatment they received in addition to psychotherapy has also been evaluated and described. ${ }^{12}$ The aims of this study are to describe the psychotherapy component of the treatment and to analyze which components were associated with better outcomes. We have included detailed analysis of the methods used with the purpose of stimulating future treatment development and implementation. Because of the detailed level of description, it will be possible for readers to compare our treatment with treatment offered to other patient populations and to choose parts of the treatment that can supplement already implemented CBT treatment elsewhere. It further enables readers to understand and consider the various components of a standard CBT treatment and their role in treatment offered to traumatized refugees living in exile with PTSD and co-morbidities like pain, anxiety and depression.

\section{Methods}

Participants and Design

All participants were screened at the Psychiatric Trauma Clinic for Refugees in Copenhagen during the period April 2008 to June 2009. The patients were 18 years or older and suffered from a mental disorder (depression or PTSD) assessed as being related to a war-related trauma. The patients had to have received treatment of at least four months' duration including treatment with antidepressants and at least four consultations with a therapist. Patients who were in need of hospitalization in the general psychiatric system were excluded from treatment in the clinic. Patients were excluded if diagnosed with psychosis or bipolar disorder (F20-29 or F31) according to ICD-10 or if they had any substance abuse apart from the regular use of benzodiazepines. Finally, patients were excluded if they did not have at least two outcome ratings (out of four possible) from initial assessment and at follow-up. Diagnoses of PTSD and depression were made according to the ICD-10 research criteria and all individual symptoms were recorded at the screening and checked with diagnostic algorithms. All patients also had PTSD and/ or depression according to DSM-IV. The study was conducted in accordance with the Helsinki Declaration and was approved by the Danish National Committee on Biomedical Research Ethics as well as by the Danish Data Protection Agency. 


\section{Data collection}

Outcomes and baseline data

Data collected during the initial assessment (two hours with a physician and one hour with a social worker) included self-report rating scales, a clinical assessment of the current psychiatric status and a structured interview collecting information on trauma, socioeconomic situation, previous mental health problems and treatment, current physical health problems and diagnosis according to the ICD-10 research criteria. At every consultation, treatment methods (topics in psychoeducation, dose of pharmacotherapy and CBT methods) were recorded. The same self-report ratings were completed at initial assessment and at the end of the treatment. If necessary, translation was provided during assessment and treatment consultations. All self-report questionnaires were available in the six most common languages (Arabic, Farsi, Bosnian / Serbo-Croatian, Russian, Danish and English), which addressed $86 \%$ of patients. If no translation was available, a translator gave a verbal translation from the official version in the language he/she felt most comfortable with.

\section{Symptom ratings}

The Hopkins Symptom Check List-25 (HSCL-25) and the Harvard Trauma Questionnaire (HTQ) are internationally used and validated self-report rating scales. ${ }^{12-15}$ HSCL-25 is a short version of the Symptom Checklist-90 with a focus on anxiety and depression symptoms. The first 16 questions of the symptom part (Part IV) of HTQ were used to evaluate the PTSDdiagnosis according to the ICD-10 and the DSM-IV diagnostic systems. For both scales, individual questions have a 1-4 Likert format with 4 being the highest symptom level.

\section{Quality of Life}

To assess quality of life we used the WHO-5 scale which is a widely used self-administered questionnaire with 5 questions $(0-5$ point Likert scale with 0 being the lowest score and 5 the highest). Raw scores have a range from 0 to 25 and are transformed into a scale from 0 (worst thinkable well-being) to 100 (best thinkable well-being). Thus, higher scores mean better well-being. ${ }^{16}$

\section{Level of Functioning}

The Sheehan Disability Scale (SDS) is a self-report rating scale, which assesses the level of functioning in terms of family, work and social network by using three visual analogue scales from $0-10$ with 10 being the lowest possible level of functioning. The score is usually reported as the total score of the three scales ranging from $0-30 .{ }^{17,18}$

\section{Therapist self-assessment}

At the beginning and at the end of the psychotherapeutic treatment (session 4 and

12) the psychologist responsible for the treatment of the patient evaluated his/her own performance in therapy by using the Beck \& Young Cognitive Therapy Rating Scale (CTRS). ${ }^{19}$ CTRS is used to evaluate the therapist's competences and consists of 11 items scored on a 6 point Likert type scale. It covers general therapy skills (feedback, understanding, interpersonal effectiveness, and collaboration), CBT skills (guided discovery, focus on key cognitions and behavior, strategy for change, application of CBT techniques) and structure in therapy (agenda, pacing and efficient use of time, and use of homework assignments). ${ }^{20}$ The scale has been used in previous CBT research ${ }^{20,21}$ and its psychometric properties are well-described. ${ }^{19}$ If pacing, use of homework and use of behavioral strategies were excluded, the internal consistency of 
the scale was acceptable (coefficient alpha $=$ 0.87 ). We therefore made an aggregated score of the remaining 8 items, which was used in the analysis of predictors of change in patient condition.

\section{Patient suitability ratings}

The therapist evaluated the patient's suitability for therapy at session 4 and session 12 using a Likert type scale of 1-5, where 5 is the best score. The scale comprises 6 items: motivation, mental flexibility, participation in therapy, empathy, introspection and treatment alliance. The scale has

Figure 1: Standard treatment model

$$
\begin{array}{|c|c|}
\hline \text { Week 1-2 } & \begin{array}{c}
\text { Baseline assessment } \\
\text { by physician }
\end{array} \\
\hline \text { Week 3-8 } & \begin{array}{c}
\text { Weekly adjustment of } \\
\text { pharmaco-therapy and } \\
\text { psychoeducation by } \\
\text { physician }
\end{array} \\
\hline \text { Week 9-24 } & \begin{array}{c}
\text { Monthly adjustment of } \\
\text { pharmaco-therapy and } \\
\text { psychoeducation by } \\
\text { physician }
\end{array} \\
\hline
\end{array}
$$

been adapted to various clinical settings. ${ }^{22,23}$ The items on the scale are highly correlated and consequently we calculated a total score for the 6 items and this was used in the further analysis (alpha $=0.92$ ).

\section{The intervention}

The treatment at the clinic is standardized and described in manuals which all physicians and clinical psychologists follow. A standard period of six months includes 10 consultations with a medical doctor and 16 sessions with a clinical psychologist (see Figure 1).

\section{Baseline assessment by social worker}


The standard medical treatment of the patient included the SSRI Sertraline, which was supplemented by the antidepressant Mianserin if the patients had sleeping problems for more than four days a week. Whenever possible, any other psychopharmacological treatment was reduced or terminated. In addition, the consultations include psychoeducation about the illness, the treatment, life in exile, the role of religion, sleep, life-style including relaxationexercises, physical and social activities, pain, cognitive functions, and the influence of the illness on the family. The psychotherapeutic intervention consisted of 16 sessions of Cognitive Behavioural Therapy (CBT) administered by six clinical psychologists who had all received ten days training in trauma-focused cognitive-behavioural therapy. Therapists received weekly individual and group supervision by certified supervisors with experience with traumatized refugees. Psychotherapy sessions were offered weekly, but since patients did not always show up for appointments, the time between consultations was sometimes longer. This could increase the overall treatment period and reduce the average number of consultations during a treatment course. The patients were seen at least once by a social worker during the treatment period.

\section{The psychotherapeutic treatment manual at PTF}

The psychotherapeutic treatment manual was developed between June 2008 and March 2009 and draws on evidence-based approaches to treatment of trauma and PTSD. ${ }^{10}$ It consists of CBT including elements of acceptance and commitment therapy (ACT) and mindfulness. Rather than focusing intensely on the trauma of the individual refugee, the methods in the manual encourage a focus on the patient's current situation and future development with regards to stress, anxiety and wellbeing in everyday life. Treatment can include 21 methods, described in more detail in Table 1.

Table 1: Methods included in the treatment manual

\section{Name of method}

Life line

Case formulation

Problem identification

Cognitive diamond

Thought records

Assumptions

Visualized Exposure

In vivo exposure

\section{Description}

Provides overview of main life events and their degree of distress.

Standard CBT map of problems and their cause.

Lists the patient's problems and goals for the therapy.

Links thoughts, feelings, bodily sensations and behaviour.

Is a tool for restructuring dysfunctional thoughts.

Working with basic assumptions about the self, others and the world.

The patient imagines approaching the feared person or situation.

Exposure where the patient actually performs the feared tasks. 
Interoceptive exposure

Focusing on the present

Breathing exercises

Body scan

Creative hopelessness

Control and acceptance

Defusion

Values

Committed action

Avoidance

Interpersonal skills

Relapse prevention

Homework
In trauma recall the client is confronted with his bodily sensations.

The patient momentarily frees himself from rumination.

Using breathing as an anchor to the present moment.

Moving attention from one body part another.

The patient is encouraged to accept a seemingly hopeless situation.

Different tools allowing the patient to accept their current situation.

By observing our thoughts we can distance ourselves from them.

Discovering which values create meaning and direction in our life.

The patient actively engages in action based on their values.

Discovery of the avoidance pattern and how it inhibits functioning.

Focuses on communication skills and anger management.

Sums up the methods and techniques that proved particularly useful.

Is integrated in all of the methods described above.

\section{Statistical analysis}

In addition to descriptive statistics, linear regression was used to investigate possible associations of methods used in psychotherapy with self-reported changes in the condition of the patient and scores on therapist assessments. The analysis was made in a two-step process due to the limited size of the data set: first significant predictors of treatment outcome ( $p$-value $<0.05$ ) were identified in regression models adjusting for the corresponding baseline rating. The significant variables were subsequently all included in multivariate regression models. HTQ and HSCL-25 multivariate models included the following predictors: patient suitability score, use of case formulation, use of thought records, use of values, mean number of therapy methods used, homework compliance and the total number of sessions with a psychologist. The SDS multivariate model included score on the therapist's self-evaluation, patient suitability score, use of breathing techniques, use of values, use of psychoeducation in therapy and homework compliance, whereas the WHO-5 model included use of mindfulness, thought records, relapse prevention and homework compliance. Pearson's correlation coefficients were used to evaluate the correlations between therapist assessments and outcome. Paired t-tests were used to evaluate the change over time in CTRS score and the therapists' evaluation of the patient. All analyses were made in STATA 11 (StataCorp LP, College Station, TX, USA). 


\section{Results}

At the clinic, 166 patients were screened and 85 were included in the final analysis (Figure 2).

For a description of the patients at baseline see Table 2 . Of all ratings, only HSCL-25 differed significantly, with a mean score for those patients who completed treatment of 3.2 and of 3.0 for those who did not complete the treatment $(\mathrm{p}=0.05)$, suggesting marginally less depression and anxiety symptom intensity in non-completers compared with completers.

\section{Psychotherapy}

All of the patients received manualized CBT as described above (see Table 3).

The therapy lasted on average 5.6 months and consisted of on average 13.5 therapy sessions. On average 11 of the 21 possible CBT-methods included in the manual were used with each patient and in each session the mean number of methods used was 2.4. In analyses adjusting for baseline scores there was a significant positive association between the mean number of methods used per session and

Figure 2: Inclusion

Screened N=166

Excluded with bipolar or psychosis $(\mathrm{N}=19)$ or no inclusion diagnosis $(\mathrm{N}=17)$

Fulfilled inclusion criteria $\mathrm{N}=130$

Complete data N=106
Did not have ratings $N=24$

\section{treatment $\mathbf{N}=21$}

Included

$\mathbf{N}=85$ 
Table 2: Treatment and patient background of all included patients $(N=85)$

\begin{tabular}{llll}
\hline Background & $\%$ & N \\
\hline
\end{tabular}

Health at baseline

PTSD

\section{7}

Depression (moderate or severe)

98

99

13

Describes hallucination, delusions or previous psychotic episodes at assessment

Treated physical symptoms at assessment

Untreated physical symptoms at assessment (excluding pain and headache)

Previous psychopharmacological treatment

Previous treatment including Psychotherapy

Any education

Working at assessment

Permanent resident status

Trauma

Experienced war

Been subjected to torture

Sex

Female

Male

Main Ethnic Groups

Iraqi

Iranian

Libanon/Palestinian

Others

Translator needed

Mean no. of years in Denmark

$\begin{array}{rr}14.5(2-30) & 6.4 \\ 43.4(21-57) & 8.0 \\ 22.6(4-46) & 8.9\end{array}$

Age

8.0

Mean no. of years since first trauma

Treatment

Medicines in addition to antidepressants 
Antipsychotics

\section{6} 5

Benzodiazepines

9

99

Received psychoeducation as part of treat-

ment

Mean (min-max)

Psychoeducation topics covered

Session with doctor

Total duration of treatment (months)

Total number of sessions

$5.7(0-10)$

8.7 (4-14)

$8.2(5.4-13.1)$

$22.3(11-38)$
Standard

deviation

2.3

1.7

1.5

4.2

Table 3: The use of psychotherapy components in the study

\section{Psychotherapy}

$\%$ Patients where

method was used (N)

No. of methods used (of 25)

Mean no. of methods per session

Sessions with psychologist

Duration of Psychotherapy (months)

Homework

Handed out

$99(84)$

Patient compliance 8\%)
Mean (sd)

$11.0(3.2)$

$2.4(0.9)$

13.5 (3.5)

$5.6(1.2)$

$7.9(5.0)$

75 (26)

$50(31)$

$\%$ Patients where

method was used $(\mathrm{N})$

Individualization (no. of sessions)

Life line

94 (80)

75 (64)

$1(0-4)$

Case formulation

21 (18)

$0(0-3)$

Problem identification

82 (70)

$2(0-9)$

Cognitive (no. of sessions)

99 (84)

The cognitive diamond

$92(78)$

$3(0-12)$ 


\begin{tabular}{rrr}
\hline Thought record & $80(68)$ & $2(0-13)$ \\
Assumptions & $36(31)$ & $0(0-9)$ \\
Avoidance & $85(72)$ & $3(0-14)$ \\
Exposure (no. of sessions) & $47(40)$ & $0(0-5)$ \\
Visualized exposure & $27(13)$ & $0(0-5)$ \\
In vivo exposure & $21(18)$ & $0(0-5)$ \\
Interoceptive exposure & $13(11)$ & - \\
Mindfulness (no. of sessions) & $92(78)$ & $1(0-12)$ \\
Focusing on the present & $74(63)$ & $0(0-5)$ \\
Breathing excercise & $74(63)$ & $0(0-5)$ \\
Body Scan & $12(10)$ & $1(0-7)$ \\
ACT (no. of sessions) $\quad 88(75)$ & $0(0-6)$ \\
Creative hopelessness & $38(32)$ & $2(0-11)$ \\
Control \& acceptance & $69(59)$ & $0(0-10)$ \\
Defusion & $35(30)$ & $1(0-6)$ \\
Values & $66(56)$ & $3(0-16)$ \\
Other methods (no. of sessions) & $46(39)$ & \\
Interpersonal skills & $68(58)$ & \\
Relapse prevention & & \\
Committed action & & \\
\hline
\end{tabular}

reduction in HTQ $(\beta=-0.12, p=0.03)$ and HSCL-25 ( $B=-0.17, p=0.02)$ scores. The discussion of relapse prevention at the end of the therapeutic treatment course was positively associated with an improvement in WHO score $(\beta=11.77, \mathrm{p}=0.02)$ in univariate analysis.

\section{Cognitive methods}

Only one patient received therapy without any of the four core cognitive methods (cognitive diamond, thought records, assumptions and avoidance). However, the use of the cognitive diamond or thought records once or twice is more likely to be part of psychoeducation than part of proper restructuring of thoughts and identification of behavioural patterns. Of the patients, $42 \%$ received therapy with the cognitive diamond or thought records used more than twice and for $30 \%$ of patients the therapy included the use of all four methods at least once. The four methods were typically used 2-5 times each. The $42 \%$ who had received therapy involving the use of restructuring of thoughts generally had a larger positive change on all four treatment outcomes than the remaining patients. When t-tests were performed, the change was significantly larger on HTQ (difference $=0.2$, $\mathrm{p}=0.05)$ and on WHO-5 (difference $=10.6$, $\mathrm{p}=0.03$ ). There was no significant difference in baseline score on any of the outcome measurements between the group that had received cognitive therapy using the core CBT 
methods several times and the group where the core methods had only been used sporadically. In univariate regression analysis there was a significant positive association between the use of thought records and improvement on WHO-5 ( $\beta=14.2, \mathrm{p}=0.02)$ and HTQ $(\beta=-0.31, p=0.01)$ and a positive significant association between the use of the cognitive diamond and HTQ $(\beta=-0.50, p=$ $0.01)$. There was a significant improvement in HSCL-25 ( $\beta=-0.38, p=0.03)$ with the use of thought records in multivariate analysis.

\section{Mindfulness methods}

At least one mindfulness exercise was used during therapy with $92 \%$ of the patients and focusing on the present and/or breathing exercises were used with $84 \%$. Only seven patients worked with body scan during therapy and all of those worked with all three mindfulness exercises during their therapy sessions. No information was available about the number of patients who practised mindfulness between therapy sessions. Each of the three methods was on average introduced two to three times in therapy. Mindfulness was associated with a decrease in quality of life on WHO-5 score ( $\beta=-18.24, \mathrm{p}=0.03$ ) in multivariate analysis and breathing exercises were associated with a decrease in level of functioning on SDS score $(\beta=-0.95$, $\mathrm{p}=0.03)$ in multivariate analysis. The patients receiving treatment with mindfulness did not differ in baseline scores compared to those who did not receive treatment.

\section{Acceptance and Commitment therapy methods}

ACT methods were used with $88 \%$ of the patients. On average each of the methods was used two to four times in therapy. Control-acceptance and values were used with $66-69 \%$ of patients whereas defusion and creative hopelessness was used with only $35-38 \%$ of the patients. The use of values was associated with a positive change in HTQ score $(B=-0.30, \mathrm{p}<0.01)$ and SDS score $(\beta=-1.47, \mathrm{p}<0.01)$ in univariate analysis.

\section{Exposure methods}

Exposure was used with $47 \%$ of the patients and the majority of patients were only treated with one of the three possible exposure methods. Interoceptive exposure was used the least and visualized exposure the most. Exposure was on average performed once or twice with each patient. There was no significant association between the use of exposure and change in any of the outcome measures. The use of exposure was associated with significantly lower scores on HSCL-25 part 2 (depression) and SDS.

\section{Individualisation of therapy}

Only $21 \%$ of the patients had a case formulation identifying a pattern of negative automatic thoughts and basic assumptions made and central problems to focus on in the therapy were not systematically identified for $18 \%$ of the patients. On average one to two sessions were spent on planning individualization of therapy in the beginning of treatment. Getting a case formulation was associated with an improvement of symptoms on HSCL-25 ( $\beta=-0.31, p=0.05)$ in univariate analysis. In terms of baseline outcome scores, the patients with a case formulation did not differ from those without.

\section{Homework}

Two variables were used to evaluate homework; how many patients who were given homework and the compliance with the homework given. Homework was handed out in $75 \%$ of the sessions and patient compliance with homework was $50 \%$. The patients 
who had low homework compliance had a marginally lower score on SDS at baseline when tested with linear regression $(\beta=0.01$, $p=0.04$ ). There were no significant differences with regard to baseline score between patients who were given homework and those who were not. Homework compliance was significantly associated with a marginal positive change on all outcome measures in univariate analysis, but only with a positive change on HTQ ( $\beta=-0.003, p=0.05)$ and SDS $(\beta=-0.015, \mathrm{p}=0.03)$ scores in multivariate analyses.

\section{Patient suitability ratings}

Patient suitability for therapy was evaluated for 46 patients in total. None of the six items changed significantly from the first to the second patient evaluation when tested with paired t-tests. The average score for all items was 3 (on a scale from 1-5). Patient suitability for therapy was positively associated with change on HTQ score $(\beta=-0.21, \mathrm{p}=0.03)$ in univariate analysis, and on SDS $(\beta=$ $-0.94, \mathrm{p}=0.01)$ and HSCL-25 $(\beta=-0.25, \mathrm{p}$ $=0.04$ ) scores in multivariate analysis. There was no correlation between the patient's suitability for treatment as evaluated by a psychologist and motivation evaluated at baseline assessment by a physician (Pearson correlation 0.02-0.2). However, there was a high correlation between the therapist's self-evaluation and the therapist's evaluation of the patient's suitability for therapy

(Pearson correlations $0.6-0.9$ ). There was a significant negative correlation between scores on patient suitability and bad baseline scores on SDS $(r=-0.26, p=0.02)$ and WHO-5 $(\mathrm{r}=-0.22, \mathrm{p}=0.0)$. There was a significant correlation between high score on patient suitability for therapy and change in outcome with regards to HSCL-25 ( $\beta=$ $-0.27, \mathrm{p}=0.02)$ and HTQ $(B=-0.24, \mathrm{p}=$ $0.03)$. For SDS and WHO-5 the trend was the same although these results were not significant.

\section{Therapist's self-assessment}

There was no significant change in the therapist's self-evaluation from $4^{\text {th }}$ to $12^{\text {th }}$ session when compared with paired t-test. The total score was 3.3 of 6 possible. There was, however, a significant correlation between self-evaluation score and SDS at baseline $(\mathrm{r}-0.33, \mathrm{p}<0.01)$. We also found a significant correlation between WHO-5 baseline score and self-evaluation score $(\mathrm{r}=$ $0.36, \mathrm{p}<0.01$ ). We found no correlation between changes in any of the outcome measures and the therapists' self-evaluation.

\section{Discussion}

The study shows that even in this multicultural group of patients with several co-morbid diseases and low level of functioning, CBT is promising and a large proportion of patients are able to participate actively and make homework from session to session. Therapists used on average 11 different methods during an average of 14 sessions, meaning that a new method was introduced in almost every session, which is fairly numerous considering that the majority of patients have no or limited experience with individual therapy and CBT in particular. Language barriers and cognitive deficits may further increase the need for repetition of methods. In addition to the high number of methods being used, most were only used one to three times with the optimal number of times depending on the method. We found a positive association between the use of core cognitive methods such as thought records and the cognitive diamond and all outcome measures. When these methods were used more than once or twice, the patients showed larger improvement and this seemed to be unrelated to the baseline conditions of the 
patients. Several less specifically cognitive methods were used many times but were not associated with a positive change (focusing on the present, interpersonal skills and avoidance). Therefore, it is not merely the number of times a method is being used which is important. However, "reverse causality" is a possibility if spontaneously improving patients are those who are able to cooperate with the cognitive methods.

It has been questioned whether traumatized refugees are able to do homework or if homework is only useful in a Western cultural context. ${ }^{24,25}$ However, we found a $50 \%$ compliance with homework, which is a relatively high rate and it seems to be associated with a small positive change in mental health symptoms and social functioning. In clinical settings, it has also been suggested that focus on restructuring of thoughts and more advanced CBT methods might not be appropriate for traumatized refugees because of the severity of the patients' condition and because of few psycho-social resources, including limited education and language barriers. We were unable to clearly identify a subgroup of patients who were mostly receiving supportive therapy, but it is likely that supportive therapy would involve a combination of the occasional use of CBT methods, using mindfulness when everything else fails and generally using few methods. Each of these factors was individually associated with less improvement in patient condition. Consequently, we have no indication that some of the patients will benefit more from a supportive and less structured therapy than CBT. In summary, our results indicate that the patients are able to participate in and benefit from CBT and restructuring of thoughts. Our results on the number of methods used suggest that it is even possible that a more thorough application of CBT methodology and the repeated use of each method could increase the treatment effect further.

Exposure was used much less than anticipated with visualized exposure therapy only used on $27 \%$ of the patients. In addition to this, exposure was only undertaken one to two times although the literature suggests that it should be used 7-12 times in the case of trauma exposure with PTSD patients. ${ }^{26}$ The lack of a positive outcome of the psychotherapy might reflect too little use of exposure. It is also possible that brief use of visualized exposure has led to sensitization rather than improvement. ${ }^{27}$ Many therapists explained that exposure was used less than planned in the manual because patients refused to participate due to high levels of distress, and in translated sessions, there may have been too little time for proper visualized exposure. Other researchers have, however, applied exposure with the same patient group, ${ }^{28}$ and thus other factors may be involved, such as a hesitation on the part of the therapist to use exposure. Mindfulness research is generally involves more intensive treatments than ours, and it is believed that the amount of daily practice by the patient is important for clinical effects. ${ }^{29,30}$ It is therefore questionable whether the use of breathing exercises a few times during therapy can be expected to have any effect. Indeed, mindfulness such as breathing may have been used when other CBT techniques were not possible. This might explain why breathing exercises and mindfulness were associated with a negative change in quality of life and level of functioning. It is also possible that mindfulness is harmful in traumatized patients suffering from PTSD as it may increase dissociation.

Several factors may influence effectiveness of therapy sessions. Few patients got a case formulation and in some cases central focus problems for the therapy were not 
identified, despite the fact that these two methods are very important for targeting the therapy to the patients' individual problems. The lack of a case formulation is likely to reflect that many patients were unable to cooperate with systematic identification of patterns of negative automatic thoughts and basic assumptions to a degree where a meaningful case formulation could be made. If this was the case, the lack of case formulations and systematic problem identification may reflect the patients' limited ability to participate in structured CBT treatment. In addition, the therapy was developed to treat PTSD and had less focus on depression, somatisation, chronic pain and other urgent problems frequently experienced by patients.

The therapists' self-evaluations were not associated with treatment results. Therapists on average rated themselves 3 out of 5 and this may reflect limited experience with this patient group and CBT.

The importance of the therapists' competence in CBT is debated, ${ }^{21}$ but is likely to be relevant in this context since studies of depressed patients have demonstrated that the more complicated and chronic the problems of the patients are, and the more anxious they are, the greater is the importance of the therapists' skills. The analysis of the therapist's evaluation of patient suitability for therapy suggests evaluation of the patient's suitability for therapy might be a useful tool in clinical contexts.

There is very limited evidence of treatment effect of psychotherapy for traumatized refugees, and the treatment models which have been evaluated are not always well-described in the literature. However, we have demonstrated that it is possible to use a thorough registration of therapy methods, which enables evaluation and development of psychotherapeutic methods for traumatized refugees.

Any comparison of psychotherapeutic treatments requires that the samples are comparable with regard to inclusion diagnosis, co-morbidities, socio-economic and cultural background. The few available studies of refugee populations differ in many respects and sufficient information about background and mental health state is not always available. Some of the studies use randomized trials whereas others are follow-up studies like the present study.

The study has important limitations which affect the conclusions that can be drawn from the data. Firstly, the lack of a control group makes it impossible to rule out the notion that the observed changes are due to spontaneous changes in the condition of the patients. Secondly, patients were treated with a combination of antidepressants, psychotherapy and psychoeducation. The observed changes may therefore be due to the effect of medication and not the effect of psychotherapy. On the other hand, the association between the use of systematic CBT and better outcome observed in this study can hardly be explained by the use of medication. Finally, the study would have benefitted from the use of blinded observerratings in addition to self-ratings. Despite methodological limitations, this study identifies clear possibilities for improvement in the described therapy and there is no indication in our data that multi-cultural patients cannot relate to the CBT model. 


\section{References}

1. Crumlish N, O'Rourke K. A systematic review of treatments for post-traumatic stress disorder among refugees and asylum-seekers. J Nerv Ment Dis. 2010 Apr;198(4):237-51.

2. Paunovic N, Ost LG. Cognitive-behavior therapy vs exposure therapy in the treatment of PTSD in refugees. Behav Res Ther. 2001 Oct;39(10):1183-97.

3. Basoglu M, Salcioglu E, Livanou M, Kalender D, Acar G. Single-session behavioral treatment of earthquake-related posttraumatic stress disorder: a randomized waiting list controlled trial. J Trauma Stress. 2005 Feb;18(1):1-11.

4. Hinton DE, Chhean D, Pich V, Safren SA, Hofmann SG, Pollack MH. A randomized controlled trial of cognitive-behavior therapy for Cambodian refugees with treatment-resistant PTSD and panic attacks: a cross-over design. J Trauma Stress. 2005 Dec;18(6):617-29.

5. Hinton DE, Pham T, Tran M, Safren SA, Otto MW, Pollack MH. CBT for Vietnamese refugees with treatment-resistant PTSD and panic attacks: a pilot study. J Trauma Stress. 2004 Oct;17(5):429-33.

6. Otto MW, Hinton D, Korbly NB, Chea A, Ba $\mathrm{P}$, Gershuny BS, et al. Treatment of pharmacotherapy-refractory posttraumatic stress disorder among Cambodian refugees: a pilot study of combination treatment with cognitive-behavior therapy vs sertraline alone. Behav Res Ther. 2003 Nov;41(11):1271-6.

7. Neuner F, Kurreck S, Ruf M, Odenwald M, Elbert T, Schauer M. Can asylum-seekers with posttraumatic stress disorder be successfully treated? A randomized controlled pilot study. Cogn Behav Ther. 2010;39(2):81-91.

8. Neuner F, Onyut PL, Ertl V, Odenwald M, Schauer E, Elbert T. Treatment of posttraumatic stress disorder by trained lay counselors in an African refugee settlement: a randomized controlled trial. J Consult Clin Psychol. 2008 Aug;76(4):686-94.

9. Neuner F, Schauer M, Klaschik C, Karunakara U, Elbert T. A comparison of narrative exposure therapy, supportive counseling, and psychoeducation for treating posttraumatic stress disorder in an african refugee settlement. JConsult ClinPsychol. 2004;72 (4):57987.

10. Bisson J, Andrew M. Psychological treatment of post-traumatic stress disorder (PTSD). Cochrane Database Syst Rev. 2007 Jul 18;(3).

11. Buhmann CB, Mortensen, EL, Ryberg J, Nordentoft M, Ekstroem M. Follow-up study of the treatment outcomes at a psychiatric trauma clinic for refugees. Torture. 2015;25(1): pp.

12. Mollica RF, Caspi-Yavin Y, Bollini P, Truong T, Tor S, Lavelle J. The Harvard Trauma Questionnaire. Validating a cross-cultural instrument for measuring torture, trauma, and posttraumatic stress disorder in Indochinese refugees. J Nerv Ment Dis. 1992 Feb;180(2):111-6.

13. Mollica RF, Wyshak G, de Marneffe D, Khuon F, Lavelle J. Indochinese versions of the Hopkins Symptom Checklist-25: a screening instrument for the psychiatric care of refugees. Am J Psychiatry. 1987 Apr;144(4):497-500.

14. Oruc L, Kapetanovic A, Culhane M, Lavelle J, Miley K, Forstbauer S, et al. Screening for PTSD and depression in Bosnia and Herzegovina: validating the Harvard Trauma Questionnaire and the Hopkins Symptom Cheklist. Int J Culture Ment Health. 2008 Dec;1(2):105-116.

15. Kleijn WC, Hovens JE, Rodenburg JJ. Posttraumatic stress symptoms in refugees: assessments with the Harvard Trauma Questionnaire and the Hopkins symptom Checklist-25 in different languages. Psychol Rep. 2001 Apr;88(2):527-32.

16. WHO. Info package; mastering depression in primary care Frederiksborg, Denmark: World Health Organisation, Regional Office for Europe, Psychiatric Research Unit; 1998 [updated 1998]. Available from: http://www.who-5.org.

17. Sheehan $\mathrm{KH}$, Sheehan DV. Assessing treatment effects in clinical trials with the discan metric of the Sheehan Disability Scale. Int Clin Psychopharmacol. 2008 Mar;23(2):70-83.

18. Lam RW, Michalak EE, Swinson RP. Assessment scales in depression, mania and anxiety. London: Taylor \& Francis; 2005. p. 152-3.

19. Vallis TM, Shaw BF, Dobson KS. The Cognitive Therapy Scale: psychometric properties. J Consult Clin Psychol. 1986 Jun;54(3):381-5.

20. Simons AD, Padesky CA, Montemarano J, Lewis CC, Murakami J, Lamb K, et al. Training and dissemination of cognitive behavior therapy for depression in adults: a preliminary examination of therapist competence and client outcomes. J Consult Clin Psychol. 2010 Oct;78(5):751-6.

21. Shaw BF, Elkin I, Yamaguchi J, Olmsted M, Vallis TM, Dobson KS, et al. Therapist competence ratings in relation to clinical outcome in cognitive therapy of depression. J Consult Clin Psychol. 1999 Dec;67(6):837-46.

22. Valbak K. Suitability for psychoanalytic psychotherapy: a review. Acta Psychiatr Scand. 2004 Mar;109(3):164-78.

23. Valbak K, Rosenbaum B, Hougaard E. Suitability for psychoanalytic psychotherapy: validation of 
the Dynamic Assessment Interview (DAI). Acta Psychiatr Scand. 2004 Mar;109(3):179-86.

24. Kinzie JD. Psychotherapy for massively traumatized refugees: the therapist variable. Am J Psychother. 2001;55(4):475-90.

25. Kinzie JD. A cross-cultural treatment of PTSD. In: Wilson JP, Friedman M, Lindy JD, editors. Treating Psychological Trauma and PTSD. New York: The Guilford Press; 2001.

26. Foa EB, Hembree EA, Rothbaum BO. Prolonged Exposure Therapy for PTSD: Emotional Processing of Traumatic Experiences, Therapist Guide. Oxford: Oxford University Press; 2007.

27. Pitman RK, Altman B, Greenwald E, Longpre RE, Macklin ML, Poire RE, et al. Psychiatric complications during flooding therapy for post- traumatic stress disorder. J Clin Psychiatry. 1991 Jan;52(1):17-20.

28. Drozdek B, Bolwek N. Evaluation of group therapy with traumatized asylum seekers and refugees - the Den Bosch Model. Traumatology. 2010 Dec;16(4):117-27.

29. Toneatto T, Nguyen L. Does mindfulness meditation improve anxiety and mood symptoms? A review of the controlled research. Can J Psychiatry. 2007 Apr;52(4):260-6.

30. Fjorback LO, Arendt M, Ornbøl E, Fink P, Walach H. Mindfulness-based stress reduction and mindfulness-based cognitive therapy: a systematic review of randomized controlled trials. Acta Psychiatr Scand. 2011 Aug;124(2):102-19. 